\title{
Response of a flexible filament in a flowing soap film subject to a forced vibration
}

\author{
Laibing Jia, ${ }^{1,2, a)}$ Qing Xiao, ${ }^{2}$ Haijun $\mathrm{Wu},{ }^{1}$ Yanfeng Wu, ${ }^{1}$ and Xiezhen Yin ${ }^{1}$ \\ ${ }^{1}$ Department of Modern Mechanics, University of Science and Technology of China, Hefei, \\ Anhui 230027, China \\ ${ }^{2}$ Department of Naval Architecture, Ocean, and Marine Engineering, University \\ of Strathclyde, Glasgow G4 OLZ, United Kingdom
}

(Received 31 October 2014; accepted 15 January 2015; published online 28 January 2015)

\begin{abstract}
The interactions between flexible plates and fluids are important physical phenomena. A flag in wind is one of the most simplified and classical models for studying the problem. In this paper, we investigated the response of a flag in flow with an externally forced vibration by using flexible filaments and soap film. Experiments show that for a filament that is either in oscillation or stationary, the external forced vibration leads to its oscillation. A synchronization phenomenon occurs in the experiments. A small perturbation leads to a large response of flapping amplitude in response. The insight provided here is helpful to the applications in the flow control, energy harvesting, and bionic propulsion areas. c 2015 AIP Publishing LLC. [http://dx.doi.org/10.1063/1.4906799]
\end{abstract}

\section{INTRODUCTION}

The interactions between a flexible plate and its ambient fluid are common phenomena in our daily life and industry, such as a flag flapping in the wind, ${ }^{1}$ snoring caused by the soft palate in human airway, ${ }^{2}$ and the flutter of a newspaper during its printing process. ${ }^{3}$ These phenomena occur when a flexible plate structure loses its instability in flow. In recent years, extracting wind or hydraulic energy from a flexible membrane vibration has become a new way to harvest renewable energy. ${ }^{4,5}$ Due to its theoretical and practical significance, the flapping of a plate in fluid flow has been investigated extensively both theoretically and experimentally. ${ }^{6}$ The most simplified and classical model for this problem is a flexible flag in a steady fluid stream. Experimental studies on the flapping behavior of a flag have been conducted in soap films, water tunnels, and low-speed wind tunnels. ${ }^{1,7,8}$

By placing flexible filaments in a flowing soap film, Zhang et al. ${ }^{1}$ studied the model of onedimensional flags in a two-dimensional wind. Their experimental observations showed that an initiallystationaryfilamentstarteditsflappingmotionwhenbothitslengthandtheexternalflowspeed exceeded some threshold values. A further study by Shelley, Vandenberghe, and Zhang ${ }^{7}$ showed that the instability of a flag also relied on the density ratio of the flag to fluid. They employed a temporal linear instability analysis to study the onset of the flag flapping. Their model considered the density, length, and bending stiffness of the plate and the density and velocity of the fluid. The instability was considered to be a local phenomenon, and vortex wake was not involved. Their theoretical analysis revealed that the system's instability is controlled by two dimensionless parameters, i.e., dimensionless density $(S)$ and dimensionless velocity $(U)$. Depending on the particular values of $S$ and $U$, the possible status of the filament can be either stable (without motion) or unstable (continuous flapping). A specific term, "stretched-straight state," is given to the stable state. Within an unstable regime, the filament could exhibit a periodic-one, a period-doubling, a quasi-periodic, or a chaos state, depending on the various combinations of $U$ and $S .^{8,9}$ Argentina and Mahadevan ${ }^{10}$ theoretically investigated the onset of a fluid induced flutter of a flag that accounted for vortex shedding using

$\overline{{ }^{a)} \text { Electronic mail: robinjia@ustc.edu.cn }}$ 
the Theodorsen approach. ${ }^{11}$ Their theory predicted the critical speed for the onset of the flapping as well as the frequency of the flapping. Both the analyses of Shelley, Vandenberghe, and Zhang ${ }^{7}$ and Argentina and Mahadevan ${ }^{10}$ are based on linearized theories. The onset of flag flapping is in the small amplitude regime, where a linear analysis is applicable.

Previous study showed that a fully developed flag flapping is geometrically nonlinear. ${ }^{9}$ And the flapping amplitude reaches half of its length. ${ }^{1}$ Connell and Yue ${ }^{9}$ developed a coupled fluid-structure direct simulation of the Navier-Stokes equation and a geometrically nonlinear structural equation to investigate the full nonlinear flapping of a flag, including the wake effects and variable tension in the body. Chen et al. ${ }^{8}$ developed a two-dimensional aeroelastic model of a flexible flag immersed in an inviscid flow by coupling the panel method with an inextensible flag model. They investigated the transition of a flag from a static steady state to a chaotic state. They found a linear relationship between the drag on a flag and the Strouhal number in periodic flapping states.

The studies mentioned above focus on a self-excited system, in which the flapping of a flag interacts with the vortices shedding in its wake. ${ }^{10}$ Apart from such a self-excited system, attention is also drawn to a flag's dynamic response to an imposed pre-specified motion or force applied to a flag. ${ }^{12-17}$ A study by Alben ${ }^{12}$ on a pitching flexible body at its leading edge showed that the generated thrust power has a series of resonant peaks with respect to the body's rigidity. Michelin and Smith ${ }^{13}$ examined a flexible heaving wing on its propulsive performance numerically and found that a resonance phenomenon occurs when the forced external oscillating frequency was equal to the natural frequency of the system. Further investigation by Manela ${ }^{14}$ on a thin elastic plate dynamic response to an external excitation illustrated that a resonance motion was excited at the plate's eigenmode when the forcing signal contained an eigen-frequency. The non-linear effects are addressed by Castro et al. ${ }^{15}$ experimentally and theoretically for a periodically forced cantilevered plate immersed in a still fluid. Alben et al. ${ }^{16}$ and Ramananarivo, Godoy-Diana, and Thiria ${ }^{17}$ investigated the propulsion performance of a self-propelled flag with a local actuator at its leading edge. The propulsive efficiency is found to be influenced by the flag's length, rigidity, and the dissipation of energy along the flag's body. These studies reveal that the dynamic of a flag is influenced by the external motion and force and established a system resonant when the external force frequency and system eigen-frequencies become close.

The studies of flags' flapping in flow are reminiscent of the cylinders' oscillation in flow. Williamson and Govardhan ${ }^{18,19}$ reviewed the free and forced vibration of a cylinder. In the free vibration studies of a cylinder, a rigid cylinder was mounted elastically while being restrained to move transversely to the flow. The vortex induced vibration (VIV) of the cylinder is a feedback process between the body motion and vortex motion. ${ }^{18}$ Once the frequency of the vortex shedding in the cylinder wake approaches the natural frequency of the flexible mounted rigid cylinder, the unsteady lift force causes an increased amplitude of the motion via a standard resonance phenomenon. The cylinder wake is then affected by the enhanced amplitude and forced to move at the natural frequency of the cylinder. ${ }^{20}$

For a forced vibrating cylinder, the rigid cylinder was mounted on a support arm ${ }^{21,22}$ or a transverse lead screw. ${ }^{23}$ The cylinder oscillated with a prescribed sinusoidal trajectory relative to the fluid. Numerical and experimental studies by Gopalkrishnan ${ }^{21}$ and Karniadakis and Triantafyllou ${ }^{24}$ revealed that the response of a forced vibration of a cylinder can either be in lock-in state, in which the vortex shedding process collapses onto the cylinder's forced vibration frequency, or in non-lockin state, in which the flow motion and force response contain multiple components at both the oscillation frequency and the vortex shedding frequency. Recent studies consider the forced vibration of a cylinder as one approach to understanding and predicting the vortex-induced vibration phenomena of freely vibrating bodies. ${ }^{18,19}$ Positive energy transfer from the fluid to a cylinder is required for a free vibrating cylinder in VIV. While for a forced vibrating cylinder, negative energy transfer may occur. Carberry et al. ${ }^{25}$ found that forced sinusoidal oscillations replicate many features of the VIV apart from the energy transfer. In order to simulate all the key features of the VIV within a sinusoidal forced vibration, the conditions, i.e., the motion of the cylinder, should be the same as that in a free vibration cylinder. ${ }^{26}$ Morse and Williamson ${ }^{23,26}$ compared the free and forced vibrations with matched experimental conditions. Their prediction from the measurement of a forced vibrating cylinder agrees with the measurements for an elastically mounted cylinder. 
Though both the flapping of a flag and the oscillation of a cylinder exhibit similarities, there are still some major differences between them. In the VIV, wake instability is an essential ingredient. The wake instability of a cylinder exists even when the cylinder is fixed. However, in the onset problem of a flag flapping, the study by Shelley, Vandenberghe, and Zhang ${ }^{7}$ shows that the instability of a flag can be considered as a local phenomenon without considering the effect of vortex shedding. In the forced vibration of a cylinder, the cylinder is rigid. The locomotion of the cylinder is prescribed. Only under the condition that the cylinder repeats its movement exactly the same as that in a free vibration case does it simulate the dynamics of a VIV cylinder. While in the forced vibration of a flag, the flag is flexible along its body. This feature allows part of the flag to move at the same pace as that of the forced vibration while the remaining part reacts as a combination of forced and free vibration. The locomotion of the flag is associated with the external forced vibration, the flag's natural vibration, and the vortex in the wake.

In this work, we use a flexible filament as the flexible flag and soap film as a two dimensional flow to examine the dynamic response of the flexible filament to an externally imposed vibration at two initial states, i.e., a stretched-straight state and an oscillating state. Our main objective is to identify the frequency and amplitude relations between the external forced vibration and the filament's dynamic response. Different from the numerical simulation methods used by the above mentioned studies, here experiment measurements are performed. Examinations focus on assessing whether an external perturbation would alter the system's inherent frequency and bring the system into a resonance state. Particular interest is given to the initial stretched-straight state, where the filament remains stationary before the forced vibration is applied, which was ignored by most of the existing studies.

\section{EXPERIMENTAL SETUP AND METHODS}

The experimental study was performed in a vertical soap film tunnel ${ }^{27,28}$ with a steady fluid flow speed ranging from 1.3 to $2.1 \mathrm{~m} / \mathrm{s}$. The viscosity of the soap film ranged from $2 \times 10^{-5}$ to $1.2 \times 10^{-5}$ $\mathrm{m}^{2} / \mathrm{s}$. A silk filament of $150 \mu \mathrm{m}$ diameter and $6.2 \times 10^{-10} \mathrm{~Pa} \mathrm{~m}^{4}$ bending stiffness was used in our study. The leading edge of the filament was fixed on a linear rail slide with a small casing (outer diameter $0.9 \mathrm{~mm}$ ). In order to avoid any disturbance in flow, the casing is kept a tiny distance from the soap film. The filament out of the casing is immersed in the soap film. It is bent into the film with surface tension. A wire is inserted into the casing to increase the friction between the filament and the casing and to prevent any rotation of the filament in the casing. The linear rail slide was connected to an electromagnetic vibration exciter, providing a sinusoidal forced vibration to the filament. A high speed camera (Mikrotron MC1311) working at 2004 frames per second was used to record the filament's locomotion, as shown in Figure 1.

In our study, three filament lengths, 15,20 , and $30 \mathrm{~mm}$, were used with four flow speeds, 1.5 , $1.8,2.0$, and $2.1 \mathrm{~m} / \mathrm{s}$. The corresponding Reynolds number ranged from $1.1 \times 10^{3}$ to $5.3 \times 10^{3}$. The external forced vibration frequency changed from 0 to $100 \mathrm{~Hz}$ with an incremental step of $5 \mathrm{~Hz}$. The amplitude of the external forced vibration was limited to below $5 \%$ of the filament's length. For ease of our following discussions, we define three frequencies:

$F_{\mathrm{p}}$ : the external forced vibration frequency, $f:$ the filament response frequency with external perturbation, $f_{0}$ :the filament response frequency at its initial status without the external perturbation, which is also called "the free flapping frequency."

$f_{1}, f_{2}, f_{3}, \ldots$.the primary, secondary, third, etc., frequency components of $f$ in the spectrum analysis.

The locomotion of the filaments in the flowing soap film was recorded by a high speed camera. The displacement of the filament was measured from the high speed camera images by an image processing program developed by us. ${ }^{28}$ Figure 2 shows the overlap of three cycles of the filament's locomotion with different external forcing frequencies. The trajectories of the filament's trailing edge are also given in the figure. The length of the filament $L=20 \mathrm{~mm}$ and the flow speed $V=1.8$ 


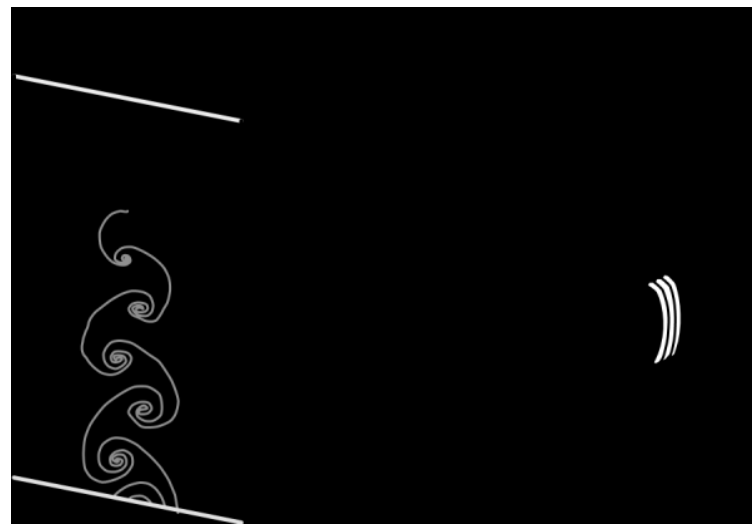

FIG. 1. Schematic diagram of the experimental setup. The leading edge of the filament is connected to a slide on a linear rail. The motion of the slide is controlled by an electromagnetic vibration exciter. The exciter is driven by an amplified sinusoidal signal with the specified frequency and amplitude.

in Figure 2(a) show the filament in a free flapping status. The locomotion of the filament is periodical. The flapping amplitude increases along the filament's body and reaches its maximum at its trailing edge. Its trailing edge moves in a figure of " 8 ." Figure 2(b) shows the filament's flapping with an external forced vibration of $F_{\mathrm{p}}=40 \mathrm{~Hz}$. With the forced vibration, the locomotion of the filament did not repeat in consequence cycles. The envelop of the filament in different cycles was not repeated. The trailing edge also shows an overlapping of several different sizes of figure " 8 ." Figure 2(c) shows the filament with another force vibration of $F_{\mathrm{p}}=45 \mathrm{~Hz}$. However, the forcing frequency only increased by $5 \mathrm{~Hz}$. The locomotion of the filament changes dramatically. And the flapping becomes periodical again. The trailing edge moves in a single figure of " $8 . "$

Figure 2 shows that with an external forced vibration, the locomotion of the filament can be quite different from that of a free flapping filament. One quantitative variable is required to describe the locomotion. Here, we selected the displacement of the filament's trailing edge to characterize the response of the filaments. Figures 3(a)-3(c) show the displacement of the filament's trailing edge transverse to the flow and their Fast Fourier Transform (FFT) result, corresponding to the flapping shown in Figures 2(a)-2(c), respectively. The displacement is non-dimensionalized by the filament's length. As we can see from Figure 3(i), the vibration of the filament's trailing edge is harmonic in free flapping status and with $F_{\mathrm{p}}=45 \mathrm{~Hz}$, while with $F_{\mathrm{p}}=40 \mathrm{~Hz}$ external forcing vibration, the flapping is tuned. Frequency analysis was then carried out to find the oscillation frequencies of the trailing edge. The result is shown in Figure 3(ii). In Figure 3(a-ii), the frequency of a free flapping filament is labeled as $f_{0}$. The positions of $f_{0}$ are marked with dotted lines in Figures 3(b-ii) and 3(cii). In Figure 3(b-ii), dominant frequency components are labeled as $f_{1}, f_{2}$, and $f_{3}$. The primary frequency component is $f_{1}=f_{0}$, the secondary frequency component is $f_{2}=F_{\mathrm{p}}$, and the third

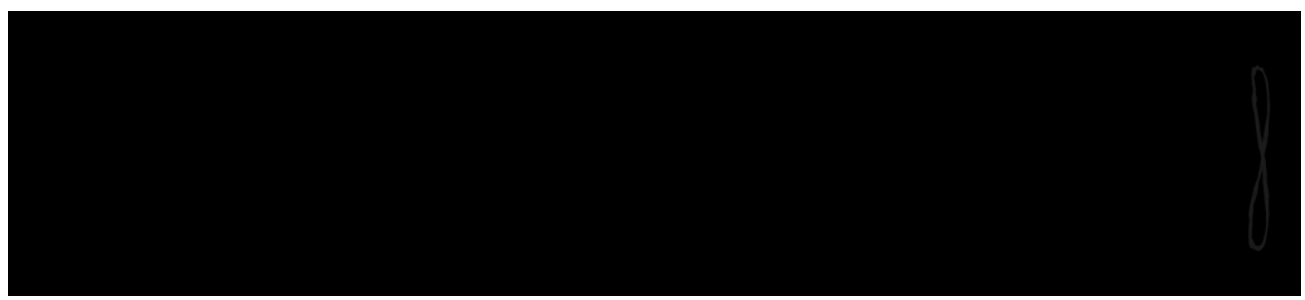

FIG. 2. Flapping of a filament with different external forced vibration frequencies. Flow speed $\mathrm{V}=1.8 \mathrm{~m} / \mathrm{s}$, filament length $\mathrm{L}=20 \mathrm{~mm}$. (a) Free flapping of the filament at a frequency $\mathrm{f}_{0}=51.9 \mathrm{~Hz}$. (b) The external forced vibration frequency $F_{p}=40 \mathrm{~Hz}$, (c) $F_{p}=45 \mathrm{~Hz}$. In each subplot, the overlapping of the filament in three cycles is shown in the center; on the right of the overlapped filaments is the trajectory of the filament's trailing edge. 


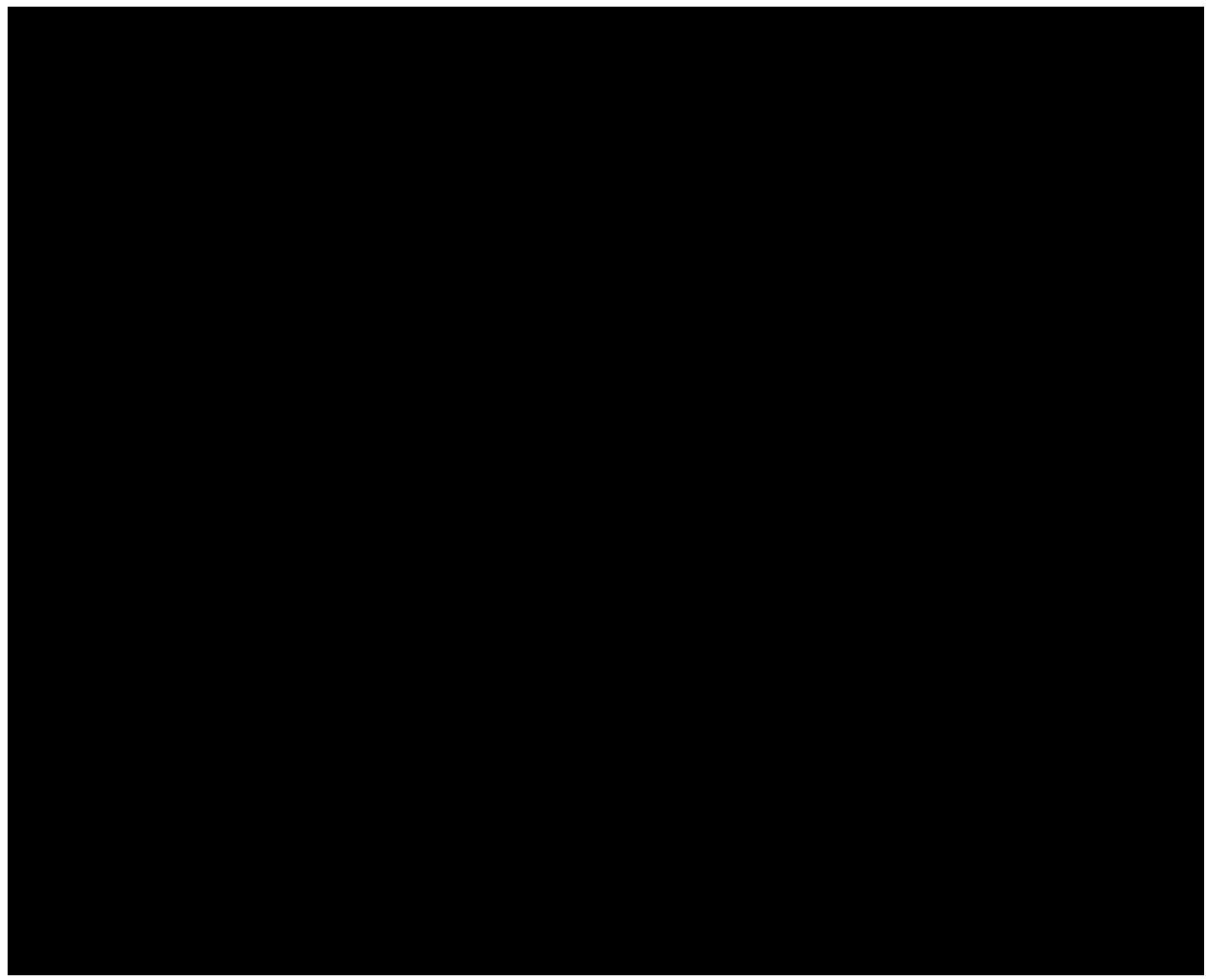

FIG. 3. Trailing edge displacement with different external forced vibration frequencies in case A. Flow speed $\mathrm{V}=1.8 \mathrm{~m} / \mathrm{s}$, filament length $\mathrm{L}=20 \mathrm{~mm}$. $\mathrm{y}^{*}$ is non-dimensionalized by $\mathrm{L}$. (i) The displacement of the trailing edge. The dots denote the experimental measurement values. The lines are fitted curves. (ii) The FFT result. The dotted line represents the position of the free flapping frequency $\mathrm{f}_{0}$. (a) The free flapping of a filament, $\mathrm{f}_{0}=51.9 \mathrm{~Hz}$. (b) The external forced vibration frequency $\mathrm{F}_{\mathrm{p}}=40 \mathrm{~Hz},(\mathrm{c}) \mathrm{F}_{\mathrm{p}}=45 \mathrm{~Hz}$.

frequency component is a combination of $f_{1}$ and $f_{2}, f_{3}=2 f_{1}-f_{2}$. In Figure 3(c-ii), the primary frequency component is $f_{1}=F_{\mathrm{p}}$.

We carried out a frequency analysis for all the experimental data of the filaments with an external forced vibration using the FFT method. The result showed that the response consisted of two major frequency components $f_{1}$ and $f_{2}$, which are equal to the free flapping frequency $f_{0}$ and forcing frequency $F_{\mathrm{p}}$. The remaining frequency components are their multiplications and combinations and they are much weaker than the primary two components. Thus, the decomposition of the trailing edge displacement,

$$
y^{*}=\sum_{i=1}^{\infty} A_{i} \sin \left(2 \pi f_{i} t+\phi_{i}\right),
$$

can be simplified into a superposition of two waves with their frequencies equal to $f_{0}$ and $F_{\mathrm{p}}$. The expression is written as

$$
\begin{aligned}
y^{*} & \approx A_{1} \sin \left(2 \pi f_{1} t+\phi_{1}\right)+A_{2} \sin \left(2 \pi f_{2} t+\phi_{2}\right) \\
& =A_{0} \sin \left(2 \pi f_{0} t+\phi_{0}\right)+A_{p} \sin \left(2 \pi F_{p} t+\phi_{p}\right),
\end{aligned}
$$

where $\mathrm{y}^{*}$ is the displacement of the trailing edge normalized by the filament length $L . A_{\mathrm{i}}, f_{\mathrm{i}}$, and $\varphi_{\mathrm{i}}$ are the amplitude, frequency, and phase of the ith component of the decomposed trailing edge displacement. $A_{0}$ is the amplitude of the free flapping frequency component $f_{0}, A_{\mathrm{p}}$ is the amplitude of the forced vibrating frequency component $F_{\mathrm{p}}$, and $\varphi_{0}$ and $\varphi_{\mathrm{p}}$ are the phases of the corresponding frequencies, respectively. 
The least squares fitting method is used to fit the measured displacements. The result is $A_{0}=0.178, \varphi_{0}=2.44, A_{\mathrm{p}}=0$ for Figure $3(\mathrm{a}), A_{0}=0.184, \varphi_{0}=4.05, A_{\mathrm{p}}=0.139, \varphi_{\mathrm{p}}=0.248$ for Figure $3(\mathrm{~b})$, and $A_{0}=0, A_{\mathrm{p}}=0.283, \varphi_{\mathrm{p}}=1.87$ for Figure 3(c). The fitted curves are plotted in Figure 3 together with the measured displacement.

To a vibration system, Strouhal number is an important parameter to describe the oscillation mechanism. The Strouhal number in our study is defined as

$$
S t=f A / V \text {, }
$$

where $f$ is the flapping frequency, $A$ is the peak to peak amplitude, and $V$ is the incoming flow velocity. In a cycle, the trailing edge of the filament moves in twice the peak to peak amplitude $A$. The numerator $f A$ can be considered as half the average speed of the filament's trailing edge $U$. The definition of Strouhal number is rewritten as

$$
S t=\frac{1}{2} \frac{\tilde{U}}{V} \text {. }
$$

The Strouhal number is the speed ratio of the structure to the fluid. The kinematic energy of the filament is expressed as

$$
E_{k \text { filament }}=\frac{1}{2} \int_{0}^{L} m_{l} u^{2}(s, t) d s,
$$

where $m_{1}$ is the linear density of the filament, $s$ is the distance along the filament's body from its leading edge, and $t$ is time. $u(s, t)$ is the velocity at position $s$ and time $t$. As we can see in Figure 2, the displacement of the filament increases from its leading edge to its trailing edge and reaches its maximum value at its trailing edge. The trajectory of the trailing edge shows that the displacement transverse to the flow is much larger than that in the flow direction. Here, we use the average speed of the filament's trailing edge $U$ as a scale of the filament's velocity. The kinematic energy integral approximates on a scale of

$$
E_{k \text { filament }} \sim m_{l} L \tilde{U}^{2} .
$$

Taking the filament length $L$ as a reference length and considering a soap film with a width $L$ passes the filament, the fluid kinematic energy is expressed as

$$
E_{k \text { fluid }}=\frac{1}{2} \rho d L L V^{2},
$$

where $\rho$ is the density of the soap film and $d$ is the thickness of the soap film. The value of $d$ depends on the soap film flow velocity.

Using Eqs. (4), (6), and (7), we get

$$
\frac{E_{k} \text { filament }}{E_{k} \text { fluid }} \sim S S t^{2},
$$

where $S=m_{1} / \rho d L$. $S$ is the dimensionless density which is a control parameter in the instability of a flag's flapping. ${ }^{7}$

For a certain filament and soap film velocity, $m_{1}, \rho, d$, and $L$ are fixed. Thus, $S$ is fixed. We get

$$
\frac{E_{k} \text { filament }}{E_{k} \text { fluid }} \sim S t^{2} .
$$

$S t^{2}$ scales the ratio of the filament's kinematic energy to the fluid's kinematic energy. Considering the external forced vibration is relatively small and only applies on the leading edge of the filament, the filament's flapping energy mainly comes from the fluid. $S t$ is an index reflecting the kinematic energy exchange between the filament and the fluid.

For a harmonic vibration like that shown in Figures 2(a) and 2(c), $f$ is equal to the only major oscillation frequency and $A$ is the peak-to-peak amplitude of the filament's trailing edge. However, for a vibration where its response contains multi-frequency components like the one shown in Figure 2(b), there are two major flapping frequencies and the amplitude of the filament's trailing edge changes from cycle to cycle. Here, the primary frequency component which has larger 
TABLE I. Free flapping frequency $\left(\mathrm{f}_{0} / \mathrm{Hz}\right)$ of filaments in flowing soap film associated with different lengths at various flow speeds. The values in brackets are sub-critical frequencies.

\begin{tabular}{lcccc} 
& \multicolumn{4}{c}{ Flow velocity $(\mathrm{m} / \mathrm{s})$} \\
\cline { 2 - 5 } Length $(\mathrm{mm})$ & 1.5 & 1.8 & 2.0 & 2.1 \\
\hline 15 & $0(33.1)$ & 64.6 & 70.1 & 77.4 \\
20 & $0(21.4)$ & 51.9 & 58.6 & 70.5 \\
30 & 32.0 & 42.7 & 54.2 & 66.3
\end{tabular}

amplitude in the two frequency components is used as $f$ in the estimation of St. For the amplitude, three candidate variables are available to describe the displacement of the trailing edge. They are the amplitude of the filament's flapping envelop, the mean of the amplitudes in different cycles, and the root mean square (RMS) of the amplitudes in different cycles. In the following study, the RMS of 50 cycles' amplitudes was used as $A$ in the calculation of $S t$. It helps to preserve $S t$ as an index of energy exchange between the filament and the fluid.

\section{RESULTS}

The dynamics response of a filament is analyzed with the deflection of the filament trailing edge. With the use of the FFT method, the relation between the external perturbation and the system response is unveiled.

Table I summarizes the experimental results for a filament's free flapping frequency $\left(f_{0}\right)$ under different flow velocities $(V)$ and filament lengths $(L)$. As seen in Table I, for the two specific parameters tested here, i.e., a filament with lengths of 15 and $20 \mathrm{~mm}$ in a flow velocity of $1.5 \mathrm{~m} / \mathrm{s}$, the filament free flapping frequency is $f_{0}=0$. The filaments are in a stretched-straight state in the flowing soap film, and no oscillation appears under these conditions. We also measured the subcritical frequency of the filament in these conditions, and details are provided in Sec. III B. Despite the above two conditions, for the remaining parameter combinations, all filaments present in oscillation states. Given a fixed filament length, its flapping frequency increases with the increase of the flow velocity. However with the same flow velocity, the filament flapping frequency decreases with the increase of its length.

In the following, we present our results based on the initial states of the filament without external perturbation. Case A focuses on the discussion about the dynamic response associated with an initially oscillating state, while case B concerns the system in an initial stretch-straight state.

\section{A. Case A}

In case A, the initial state of the filament is oscillating. External forced vibrations with different frequencies were applied to the leading edge of the filament. We tested 10 groups for filament length and flow speed combinations. The results were measured by the displacement of the filament's trailing edge. Frequency analysis of the displacement by the FFT method was carried out. We found that a synchronization phenomenon occurs in the response frequency $f$ between its two components that are equal to the forced vibration frequency $F_{\mathrm{p}}$ and the free flapping frequency $f_{0}$, respectively. This applied to all the flow velocities and filament lengths tested in this case. A general observation is that given the forced vibration frequency $F_{\mathrm{p}}$ is zero, the filament flaps at its free flapping frequency $f_{0}$. With the region of $F_{\mathrm{p}}$ being greater than 0 but far from $f_{0}$, the filament flaps in a quasi-periodic oscillation state and presents two response frequency components in $f$. For most parameters, the dominant frequency component is equal to $f_{0}$, while the secondary frequency component is equal to $F_{\mathrm{p}}$. With $F_{\mathrm{p}}$ near $f_{0}$, the synchronization phenomenon appears. The system behaves in a period-one flapping state with a single response frequency that is equal to $F_{\mathrm{p}}$.

Figure 4 shows the result of a filament with length $L=20 \mathrm{~mm}$ in case A. In Figure 4(i-iii), the flow velocities $(V)$ are $1.8,2.0$, and $2.1 \mathrm{~m} / \mathrm{s}$, respectively. The free flapping frequencies of the 


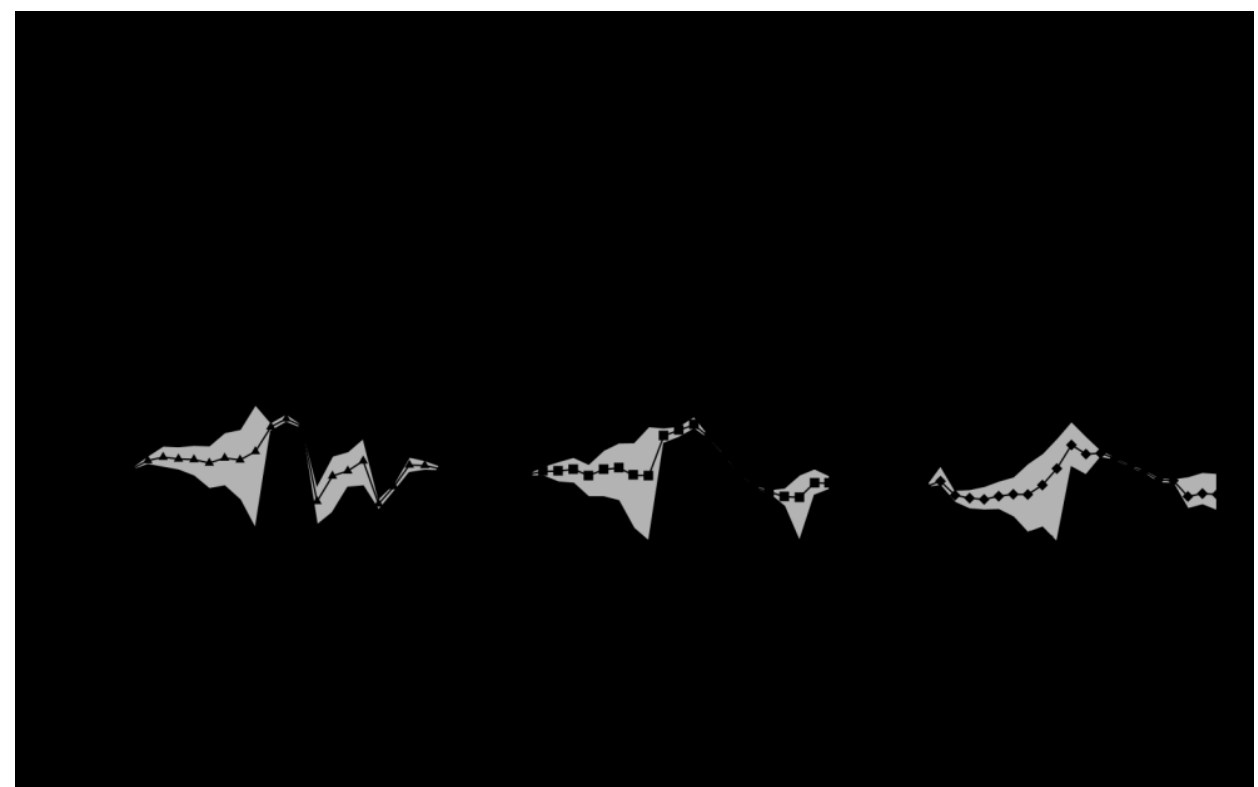

FIG. 4. Response of filament with an initial oscillating state. The length of filament $\mathrm{L}=20 \mathrm{~mm}$. (i-iii) Triangle, square, and diamond symbols represent the experimental data at the flow speed of $1.8 \mathrm{~m} / \mathrm{s}, 2.0 \mathrm{~m} / \mathrm{s}$, and $2.1 \mathrm{~m} / \mathrm{s}$, respectively. $F_{\mathrm{p}}{ }^{*}$ is the dimensionless external forced vibration frequency. (a) $\mathrm{f}^{*}$ is the dimensionless response frequency of the filament's trailing edge. Filled and opened symbols represent the primary and secondary frequency components of $\mathrm{f}^{*}$, respectively. (b) $\mathrm{A}^{*}$ is the dimensionless amplitude of the filament's trailing edge. The gray regions denote the variation ranges of the amplitudes in different cycles. The top and bottom boundaries of the gray regions are the maximum and minimum flapping amplitudes. (c) St is the Strouhal number of the filament.

filament $f_{0}$ are $51.9,58.6$, and $70.5 \mathrm{~Hz}$, respectively. In Figure 4 , the forced vibration frequency $F_{\mathrm{p}}$ and response frequency $f$ are non-dimensionalized by the free flapping frequency $\left(f_{0}\right)$ and denoted as $F_{\mathrm{p}}{ }^{*}$ and $f^{*}$.

A frequency lock-in phenomenon is clearly shown in Figure 4(a). Within the lock-in regime where $F_{\mathrm{p}}{ }^{*}$ is around one, the response frequency of the filament is locked to the perturbation frequency imposing on its leading edge. Out of the lock-in regime, both the perturbation frequency and the free flapping frequency are observed. In our study, the resonance is centered by the filament's free flapping frequency. This frequency lock-in phenomenon is different from the synchronization or lock-in phenomena in the free oscillation of a cylinder (VIV). In a classical synchronization phenomenon in VIV, there is a resonance between the vortex shedding frequency and the natural frequency of the elastically mounted rigid cylinder. ${ }^{18,20}$ The forcing frequency generated by vortices is locked to the cylinder's natural frequency. The resonance is centered by the cylinder's natural frequency. In both of the VIV problem and present study, the resonance regimes are centered by the structures' natural frequencies. However, in the present study, a forced vibration is applied to the leading edge of the filament externally. The external forced vibration will not be affected by the motion of the filament and fluid. In the resonance, the filament changes its flapping frequency to resonate the perturbation frequency once two frequencies become close. By contrast, the lock-in phenomenon studied here is very similar to the lock-in phenomena in the forced vibration of a cylinder. In the lock-in regime of the forced vibration of a cylinder, the vortex shedding process collapses onto the cylinder's forced vibration frequency. Here in the lock-in regime, the filament's free flapping frequency component disappears, leaving only the forced vibration frequency component.

The variation of the dimensionless amplitude $A^{*}$ with $F_{\mathrm{p}}{ }^{*}$ is shown in Figure 4(b). The amplitudes of the filament in 50 cycles were measured from the high speed camera recorded images. They are non-dimensionalized by the filament's length $L$ and denoted as $A^{*}$. In Figure 4(b), the filled triangle, rectangle, and diamond symbols represent the RMS amplitudes of the filament trailing edge. The gray regions are the variation ranges of the amplitudes in different cycles. The top and 


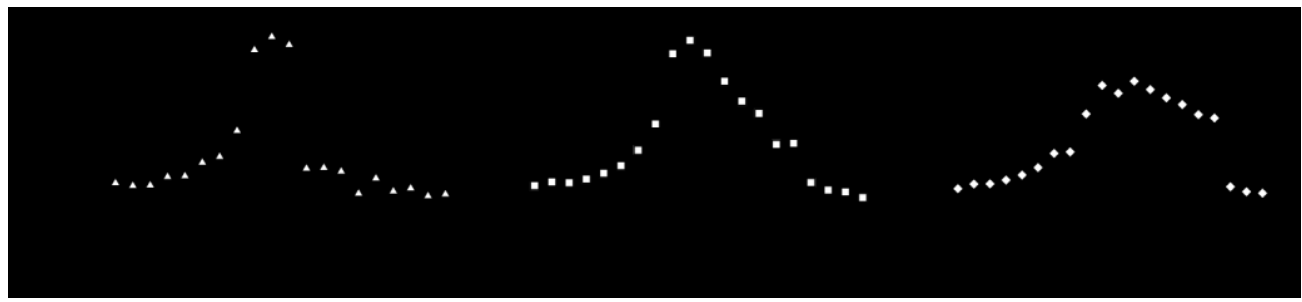

FIG. 5. Amplitudes of two response frequency components. The length of filament $\mathrm{L}=20 \mathrm{~mm}$. Triangle (a), square (b), and diamond (c) symbols represent the experimental data at the flow speed of $1.8 \mathrm{~m} / \mathrm{s}, 2.0 \mathrm{~m} / \mathrm{s}$, and $2.1 \mathrm{~m} / \mathrm{s}$, respectively. Filled and opened symbols denote the amplitudes $A_{0}$ and $A_{p}$, respectively. $F_{p}{ }^{*}$ is the dimensionless external forced vibration frequency. $\mathrm{A}^{*}$ is dimensionless amplitude.

bottom boundaries of the gray regions dedicate the maximum and minimum flapping amplitudes for the filament at the corresponding external forced vibration. A large gray zone means the flapping is tuned like the one shown in Figure 3(b), while a narrow gray zone means the flapping is almost sinuous with constant amplitude.

Due to the external force vibration applied to the leading edge of the filament, the amplitude at the filament's trailing edge is changed. Within a range close to the filament's free flapping frequency, the amplitude is enhanced. The maximum RMS amplitude reaches 0.61 times the filament length or 1.74 times the initial free flapping amplitude. In the experiment, the maximum amplitude was not always observed at the center in the lock-in frequency $(F \quad 1)$ as shown in Figures $4(\mathrm{~b}-\mathrm{i})$ and $4(\mathrm{~b}-$ ii). Sometimes it is observed outside the range, as shown in Figure 4(b-iii).

The displacement of the trailing edge is described in Eq. (2). It is a superposition of two waves. We calculated the amplitudes of two frequency components $A_{0}$ and $A_{\mathrm{p}}$ to investigate their contributions to the amplitude. Figure 5 shows the variations of $A_{0}$ and $A_{\mathrm{p}} . A_{\mathrm{p}}$ increases as the external forcing frequency increases to approach the free flapping frequency $f_{0}$. It reaches its peak at $F$ 833 , respectively. It then decreases almost linearly until the filament exits the lock-in status. It continuously decreases as the increase of $F$ remains around a certain value outside of the lock-in regime. Since the displacement of the filament's trailing edge is limited, the value of $A_{0}$ is suppressed with the increase of $A_{\mathrm{p}}$. In the lock-in regime, it becomes 0 . In Figure 5(c), which corresponds to Figure 4(b-iii), $A_{\mathrm{p}}$ has already increased to its maximum at $f^{*}=0.694$ but $A_{0}$ has not been suppressed. The superposition of two waves creates the maximum amplitude outside the lock-in regime.

The variation of Strouhal number is shown in Figure 4(c). Given a filament length of $20 \mathrm{~mm}$, the $S t$ numbers without perturbation are all around 0.2, irrespective of the flow speeds. With an external perturbation, the $S t$ numbers vary between 0.11 and 0.35 for most of the tests. Though the amplitude of the trailing edge may not center around $F_{\mathrm{P}^{*}}=1$, the maximum $S t$ number centers around $F_{\mathrm{P}^{*}}=1$. This suggests that the lock-in phenomenon leads to a large energy exchange between the structure and fluid. In addition, with some $F_{\mathrm{p}}{ }^{*}$, the $S t$ becomes lower than that of a free flapping filament, which indicates that the external perturbation suppresses the energy exchange between the structure and fluid.

\section{B. Case B}

In case $\mathrm{A}$, the initial state of the filament is stationary, i.e., the free flapping frequency of the filament $f_{0}=0$. When a perturbation is applied (i.e., $F_{\mathrm{p}}>0$ ), the system exhibits a forced vibration state. The dominant response frequency component is equal to $F_{\mathrm{p}}$. However, we found an additional frequency component when the external forcing frequency $F_{\mathrm{p}}$ is low. The additional frequency component did not present without the external forced vibrations. It is independent from the forced vibration amplitude as well as the frequency.

Following the study of Marais et al. ${ }^{29}$ on the impulse response of a cylinder wake below the critical Reynolds number of the Bénard-von Kármán instability, we tested the subcritical response 


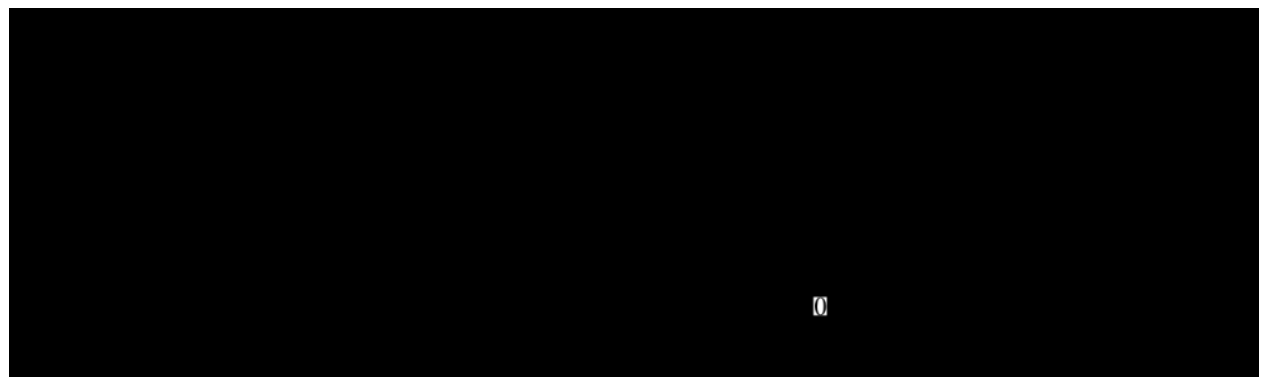

FIG. 6. Trailing edge displacement with an impulsive motion applied to the leading edge of a filament. $\mathrm{L}=20 \mathrm{~mm}, \mathrm{~V}=1.5$ $\mathrm{m} / \mathrm{s}$. (a) The displacement of the trailing edge. (b) The FFT result. The sub-critical frequency is marked as $\mathrm{f}_{0}$ (sub).

of the filament in a stretched-straight state by applying an impulsive perturbation to the leading edge of the filament. Once the impulsive motion was applied, the filament started to flap. The flapping amplitude reduced as time passed by. After several flapping cycles, the filament restored its stretched-straight state. By analyzing the induced motion of the filament's trailing edge, we obtained the sub-critical frequency for the filament. Figure 6 shows the displacement of a filament's trailing edge and its FFT result. The length of filament $L=20 \mathrm{~mm}$ and the flow velocity $V=1.5 \mathrm{~m} / \mathrm{s}$. As we can see in Figure 6(a), once the filament starts to flap, the amplitude of the filament's trailing edge increases and then decreases until the filament rests. The FFT result of the sub-critical response is marked as $f_{0}(\mathrm{sub})$ in Figure 6(b): $f_{0}(\mathrm{sub})=21.4 \mathrm{~Hz}$. The steps in the waveform are due to the resolution of the images captured during the test. In Table I, the values in brackets are sub-critical frequencies for the filament with lengths of 15 and $20 \mathrm{~mm}$ at a flow speed of $1.5 \mathrm{~m} / \mathrm{s}$.

We found that the secondary response frequency component is equal to the sub-critical frequency. In case B, the initial state of the system is stable. With an external forced perturbation, external energy is imported into the system, which brings the system out of its neutral stability state. The existence of a sub-critical frequency component shows the system stability breakdown.

Another finding is related to the induced flapping amplitude. We found that within a certain range of forced vibration frequency, a small external forced vibration at the filament leading edge can lead to large flapping amplitude at its trailing edge. This phenomenon is distinguished from the hysteresis discovered by Zhang et al., ${ }^{1}$ where the flapping status remains even when the external force disappears. However, in the current case, the filament restores the stretched-straight state once the external forced vibration is released.

Figure 7 shows the displacement of the filament's trailing edge and its FFT result. The forced vibration amplitude herein is 5\%L. The dotted line in Figure 7(iii) denotes the position of the subcritical frequency. Figure $7(\mathrm{a})$ is the result with an external forcing frequency $F_{\mathrm{p}}=15 \mathrm{~Hz}$. In Figure 7(a-iii), four peaks are observed from the FFT analysis: $f_{1}=F_{\mathrm{p}}, f_{2}=f_{0}(\mathrm{sub}), f_{3}=2 f_{1}$, and $f_{4}=3 f_{1}$. In contrast to Figure 7(a-iii), the result with an external forcing frequency $F_{\mathrm{p}}=40 \mathrm{~Hz}$ shown in Figure 7(b-iii) indicates the existence of only one frequency component, i.e., $f_{1}=F_{\mathrm{p}}$, while the frequency component that is equal to the subcritical frequency disappears. The flapping of the filament in Figure 7(b-i) is similar to that in Figures 2(a) and 2(c).

We tested periodic forced vibrations with amplitudes of $3 \%, 4 \%$, and $5 \%$ of the filament lengths $(15$ and $20 \mathrm{~mm})$ at flow speed of $1.5 \mathrm{~m} / \mathrm{s}$, respectively. All of the experimental results confirm the existence of the sub-critical frequency component at a low forced vibration frequency and the enhancement of flapping amplitude.

Figure 8 shows some results for case B. The length of the filament $L=20 \mathrm{~mm}$ and the flow velocity $V=1.5 \mathrm{~m} / \mathrm{s}$. Since the free flapping frequency of the filament $f_{0}=0 \mathrm{~Hz}$ for case B, the subcritical frequency is used to non-dimensionalize the forced vibration frequency $F_{\mathrm{p}}$ and response frequency $f$ in Figure 8. The amplitude is non-dimensionalized by $L$, and $S t$ is estimated using the primary component of $f$.

In Figure 8(a), a secondary frequency component appears where $F_{\mathrm{p}}{ }^{*}$ ranges from 0.23 to 0.93 . Given different forced vibration amplitudes, this secondary frequency only varies a little. Unlike 


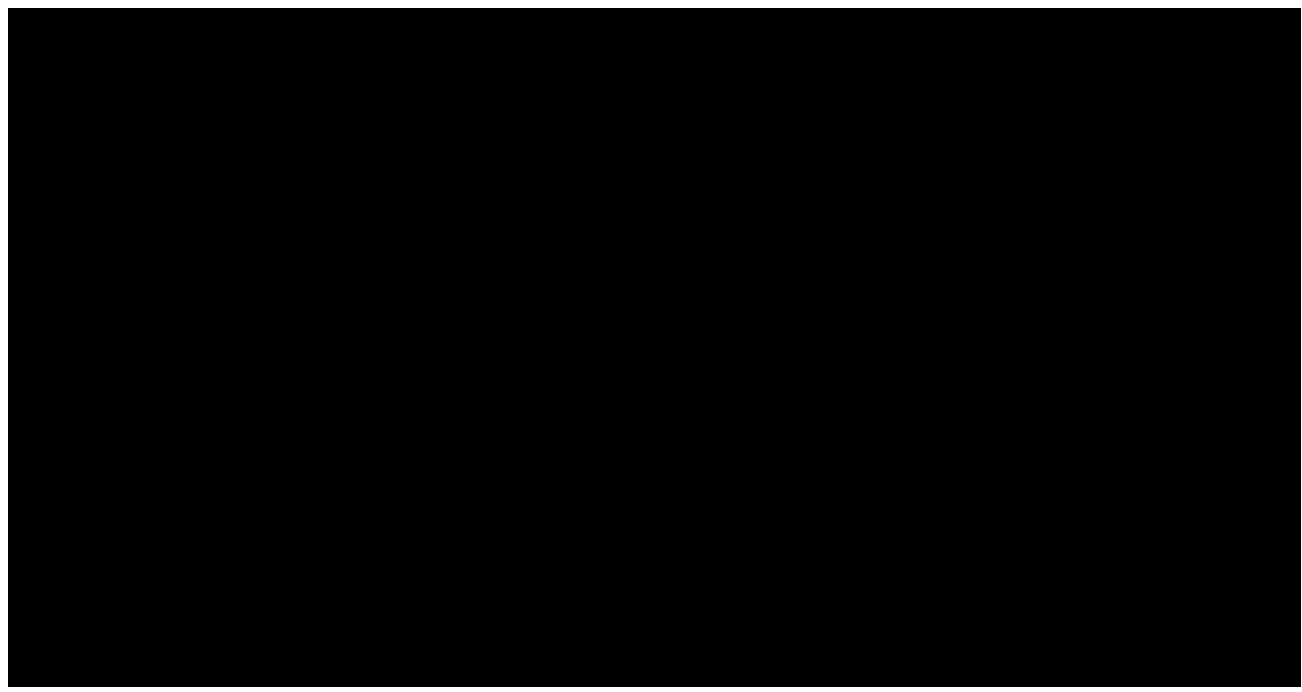

FIG. 7. The flapping of a filament at different external forced vibration frequencies in case $\mathrm{B}$. $\mathrm{L}=20 \mathrm{~mm}, \mathrm{~V}=1.5 \mathrm{~m} / \mathrm{s}$, the forced vibration amplitude is 5\%L. (i) The overlapping of the filament in three cycles. (ii) Trailing edge displacement with different external forced vibration frequencies. (iii) The FFT result. The dotted line represents the position of the sub-critical flapping frequency $\mathrm{f}_{0}$ (sub). (a) $\mathrm{F}_{\mathrm{p}}=15 \mathrm{~Hz}$, (b) $\mathrm{F}_{\mathrm{p}}=40 \mathrm{~Hz}$.

case $\mathrm{A}$, here the frequency component which is equal to the forced vibration frequency $F_{\mathrm{p}}$ is always dominant. The flapping amplitude shown in Figure 8(b) presents an increased trend of $A^{*}$ for $F$ 3, mainly in a range between 0.93 and 2.1. Consequently, the St number also increases with the amplitude. At $F_{\mathrm{p}}$ around 1.9, the $S t$ number is around 0.2 , which is close to the $S t$ number of the free flapping filament in an oscillation state.

The amplitude enhancement is limited to a range of forced vibration frequencies. In order to analyze this phenomenon, we studied the vortex structure in the filament wake. Though the wake is not a key factor in the onset of a filament's flapping, it plays a role in the full developed nonlinear flapping of a filament. According to the Vorticity Moment Theory (VMT) proposed by $\mathrm{Wu},{ }^{30}$ for an airfoil moving in a fluid in a two-dimensional plane, the lift force per unit length of a airfoil span is

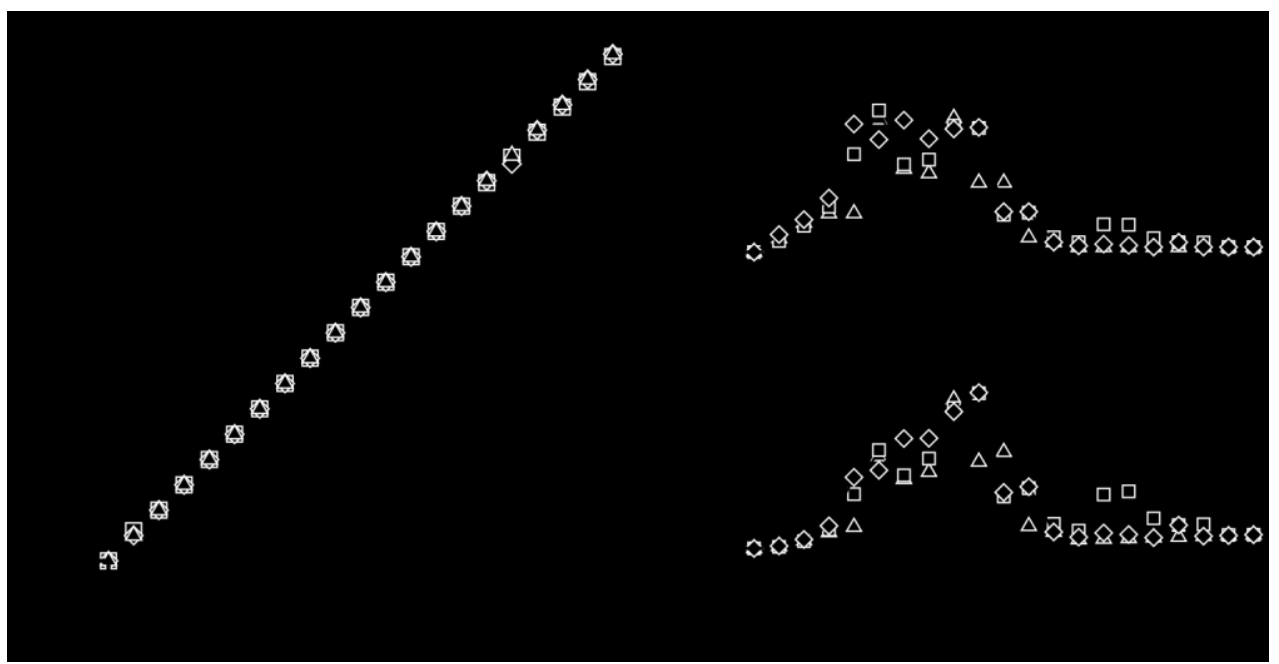

FIG. 8. Response in the stretched-straight state. The length of filament $\mathrm{L}=20$. Triangle, square, and diamond symbols represent the experimental data at forced vibration amplitudes of 3\%, 4\%, and 5\% of L, respectively. (a) Filled and opened symbols represent the primary and secondary frequencies, respectively. (b) A* is the dimensionless amplitude of the filament's trailing edge. (c) St is the Strouhal number of the filament.

given by 


$$
F_{L}=\rho \frac{d}{d t} \int_{R_{f}} \int x \omega d x d y,
$$

where $x$, y are coordinates defining the flow plane, $x$ is the flow direction, $\rho$ is the fluid density, $R_{\mathrm{f}}$ is the fluid region, and $\omega$ is the vorticity. The equation provides an estimation of the lift acting on the filament. The force relies on the strength of the vortices and their distance downstream. In the far field from the foil, vortices are presented in pairs with opposite rotating directions. The lift generated by them is canceled by each other. This leaves only the lift generated by the most recently shed vortices, i.e., the lift is determined by the last shed vortices and their moving speed and distance from the filament. Considering the effect of forced vibration at the filament leading edge, the filament flaps at $F_{\mathrm{p}}$; therefore, the redistribution of flow energy follows the frequency of $F_{\mathrm{p}}$. The continuously up and down flapping motion redistributes the flow energy associated with vortices and determines their strength. At a low forced vibration frequency $F_{\mathrm{p}}$, the locomotion of the filament is too weak to produce sufficiently concentrated vortices. At a high forced vibration frequency, the flow energy is redistributed into too many vortices. For each vortex, its strength is also weak. In both conditions, the strength of the vortices is too weak to maintain large amplitude flapping for the filament.

In Figure 9, three snapshots of a filament's flapping at forced vibration frequencies of $10 \mathrm{~Hz}$, $40 \mathrm{~Hz}$, and $75 \mathrm{~Hz}$ are given. As seen from Figure 9(a), where $F_{\mathrm{p}}=10 \mathrm{~Hz}$, the vortices are so weak that they are almost invisible. When $F_{\mathrm{p}}$ is increased to $40 \mathrm{~Hz}$ in Figure 9(b), the flapping amplitude becomes large and a strong vortices structure is generated, as evident from Figure 9(b). Under this situation, a large oscillating amplitude leads to a strong vortex, which is able to retain a large oscillating amplitude. When the forced vibration frequency is further increased to $75 \mathrm{~Hz}$ as shown in Figure 9(c), the vortex in the wake appears to be too weak to interact with the filament, and the filament tends to be stable and is unable to develop a large amplitude flapping.

\section{DISCUSSION}

In the present study, we examined the dynamic response of a flexible filament to an external forced vibration in a flowing soap film with its initial state either in an oscillating (case A) or a

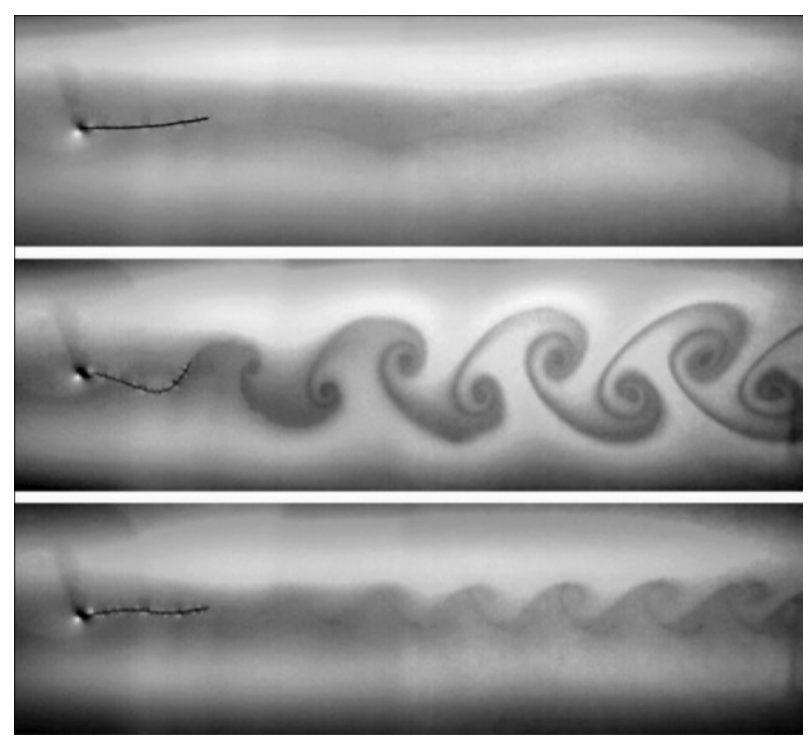

FIG. 9. Snapshots of flag flapping at different forced vibration frequencies for a stretched-straight case. The filament length $\mathrm{L}=20 \mathrm{~mm}$, flow speed $\mathrm{V}=1.5 \mathrm{~m} / \mathrm{s}$, the forced vibration amplitude is $5 \% \mathrm{~L}$. (a) The forced vibration frequency is $10 \mathrm{~Hz}$, (b) the forced vibration frequency is $40 \mathrm{~Hz}$, (c) the forced vibration frequency is $75 \mathrm{~Hz}$. 
stretched-straight state (case B). In an oscillating state, the study shows a synchronization phenomenon that the system oscillates at the same frequency as that of the external forced vibration. For an initial stretched-straight state, the system presents another frequency component in addition to the forced vibration frequency. With an initial oscillating state, the forced vibration at the leading edge leads to an increase in the trailing edge oscillating amplitude and $S t$ number in the lock-in regime. In a stretch-straight state, the results show that with some parameters, the $S t$ number of the filament is equal to that in the oscillation state.

St number represents a ratio between the trailing edge velocity and fluid velocity. Though, its value cannot be used to quantitatively measure the kinetic energy exchange between fluid and structure. It provides a qualitative way to evaluate the energy exchange. The extent of the extracted energy for a flexible structure from its surrounded fluid relies on its structural deformation. By forcing the leading edge to vibrate with small oscillating amplitude, our study on a flexible filament dynamic response shows that a small amount of energy input into this system can trigger and even enhance the system instability, which allows the structure to extract more energy from fluid. The periodic disturbance plays a "bringing a heavy fist out of a light one" role in the system energy extraction. This idea could be beneficial for exploring and designing some renewable energy devices based on a flexible oscillating motion.

The experimental setting in the present study is similar to the studies on a forced vibrating cylinder. ${ }^{21,24}$ In these studies, a lock-in regime was found in the middle of two non-lock-in regimes. In the non-lock-in state, the dominant frequency component in the wake of the cylinder is equal to the wake shedding frequency without forcing. The active control method was found to be very effective in the reinforcement of the vortex street in the wake of the cylinder. Similar results were observed in the present study. Specifically, in case A, the lock-in regime was found between two non-lock-in regimes. The free vibration frequency was dominated when the external forced vibration frequency was far away from the filament's free flapping frequency. However, when the forced vibration frequency became close to the lock-in regime, the forced vibration frequency was dominant. Moreover, in case B, the forced vibration frequency component always dominated in the response. Our study also shows the effectiveness of an active vibration control to enhance the flapping of a filament. In the existing studies of a forced vibrating cylinder ${ }^{23,26}$ lift and drag were commonly compared with that of a free vibrating cylinder. However in our study on the forced vibration of a flag using a filament in a soap film, the force measurement is hard to perform, especially with the unsteady movement of the filament's leading edge. The comparison of forces in the free and forced flapping of a flag requires further studies with water tunnel experiments and numerical simulations.

\section{ACKNOWLEDGMENTS}

This work is supported by the NSFC (Grant Nos. 11372305 and 11002138) and K. C. Wong Education Foundation for a Royal Society K. C. Wong Postdoctoral Fellowship. We appreciate the valuable suggestions from the referees.

1 J. Zhang, S. Childress, A. Libchaber, and M. Shelley, "Flexible filaments in a flowing soap film as a model for onedimensional flags in a two-dimensional wind," Nature 408, 835 (2000).

2 L. Huang, "Flutter of cantilevered plates in axial flow," J. Fluids Struct. 9, 127 (1995).

3 Y. Watanabe, S. Suzuki, M. Sugihara, and Y. Sueoka, “An experimental study of paper flutter,” J. Fluids Struct. 16, 529 (2002).

4 J. J. Allen and A. J. Smits, "Energy harvesting ell," J. Fluids Struct. 15, 629 (2001).

5 S. Michelin and O. Doaré, "Energy harvesting efficiency of piezoelectric flags in axial flows," J. Fluid Mech. 714 , 489 (2013).

6 M. J. Shelley and J. Zhang, "Flapping and bending bodies interacting with fluid flows," Annu. Rev. Fluid Mech. 43 , 449 (2011).

7 M. Shelley, N. Vandenberghe, and J. Zhang, "Heavy flags undergo spontaneous oscillations in flowing water," Phys. Rev. Lett. 94, 094302 (2005).

8 M. Chen, L. Jia, Y. Wu, X. Yin, and Y. Ma, "Bifurcation and chaos of a flag in an inviscid flow," J. Fluids Struct. 45, 124 (2014).

9 B. S. H. Connell and D. K. P. Yue, "Flapping dynamics of a flag in a uniform stream," J. Fluid Mech. 581, 33 (2007).

${ }^{10}$ M. Argentina and L. Mahadevan, "Fluid-flow-induced flutter of a flag," Proc. Natl. Acad. Sci. U. S. A. 102, 1829 (2005).

11 T. Theodorsen, "General theory of aerodynamic instability and the mechanism of flutter," NACA Report No. 496 Technical Report, National Advisory Committee for Aeronautics, 1935. 
${ }^{12}$ S. Alben, "Optimal flexibility of a flapping appendage in an inviscid fluid," J. Fluid Mech. 614, 355 (2008).

${ }^{13}$ S. Michelin and S. G. L. Smith, "Resonance and propulsion performance of a heaving flexible wing," Phys. Fluids 21, 071902 (2009).

${ }^{14}$ A. Manela, "Vibration and sound of an elastic wing actuated at its leading edge," J. Sound Vib. 331, 638 (2012).

${ }^{15}$ R. F. A. Castro, L. Guillamot, A. Cros, and C. Eloy, "Non-linear effects on the resonant frequencies of a cantilevered plate," J. Fluids Struct. 46, 165 (2014).

${ }^{16}$ S. Alben, C. Witt, T. V. Baker, E. Anderson, and G. V. Lauder, "Dynamics of freely swimming flexible foils," Phys. Fluids 24, 051901 (2012).

${ }^{17}$ S. Ramananarivo, R. Godoy-Diana, and B. Thiria, "Passive elastic mechanism to mimic fish-muscle action in anguilliform swimming," J. R. Soc., Interface 10, 20130667 (2013).

${ }^{18}$ C. H. K. Williamson and R. Govardhan, "Vortex-induced vibrations," Annu. Rev. Fluid Mech. 36, 413 (2004).

${ }^{19}$ C. H. K. Williamson and R. Govardhan, "A brief review of recent results in vortex-induced vibrations," J. Wind Eng. Ind. Aerodyn. 96, 713 (2008).

${ }^{20}$ E. De Langre, "Frequency lock-in is caused by coupled-mode flutter," J. Fluids Struct. 22, 783 (2006).

${ }^{21}$ R. Gopalkrishnan, "Vortex-induced forces on oscillating bluff cylinders," Ph.D. thesis, Massachusetts Institute of Technology, 1993.

22 J. Carberry, J. Sheridan, and D. Rockwell, "Forces and wake modes of an oscillating cylinder," J. Fluids Struct. 15, 523 (2001).

${ }^{23}$ T. L. Morse and C. H. K. Williamson, "Prediction of vortex-induced vibration response by employing controlled motion," J. Fluid Mech. 634, 5 (2009).

${ }^{24}$ G. E. Karniadakis and G. S. Triantafyllou, "Frequency selection and asymptotic states in laminar wakes," J. Fluid Mech. 199, 441 (1989).

${ }^{25}$ J. Carberry, R. Govardhan, J. Sheridan, D. Rockwell, and C. Williamson, "Wake states and response branches of forced and freely oscillating cylinders," Eur. J. Mech., B: Fluids 23, 89 (2004).

${ }^{26}$ T. L. Morse and C. H. K. Williamson, "Employing controlled vibrations to predict fluid forces on a cylinder undergoing vortex-induced vibration,” J. Fluids Struct. 22, 877 (2006).

${ }^{27}$ M. A. Rutgers, X. L. Wu, and W. B. Daniel, "Conducting fluid dynamics experiments with vertically falling soap films," Rev. Sci. Instrum. 72, 3025 (2001).

${ }^{28}$ L. Jia, The Interaction Between Flexible Plates and Fluid in Two-dimensional Flow (Springer, 2014).

${ }^{29}$ C. Marais, R. Godoy-Diana, D. Barkley, and J. E. Wesfreid, "Convective instability in inhomogeneous media: Impulse response in the subcritical cylinder wake," Phys. Fluids 23, 014104 (2011).

$30 \mathrm{~J}$. Wu, "Theory for aerodynamic force and moment in viscous flows," AIAA J. 19, 432 (1981). 